\title{
MICROBIOLOGICAL STUDY OF A PERSPECTIVE HEPATOPROTECTIVE AGENT BASED ON DRY EXTRACT FROM PRUNUS DOMESTICA FRUITS
}

\author{
Nataliia Filimonova \\ Department of Microbiology, Virology and Immunology \\ National University of Pharmacy \\ 53 Pushkinskaya str., Kharkiv, Ukraine, 61002 \\ megiddo@ukr.net \\ Bashar Jabbar Ali Sahlanee \\ Department of Biological Chemistry \\ National University of Pharmacy \\ 53 Pushkinskaya str., Kharkiv, Ukraine, 61002 \\ citochrom@i.ua \\ Igor Senyuk \\ Department of Biological Chemistry \\ National University of Pharmacy \\ 53 Pushkinskaya str., Kharkiv, Ukraine, 61002 \\ citochrom@gmail.com \\ Alevtyna Kononenko \\ Department of Physiology and Human Anatomy \\ National University of Pharmacy \\ 53 Pushkinskaya str., Kharkiv, Ukraine, 61002 \\ alevtina19820103@gmail.com
}

\footnotetext{
Abstract

One of the main directions of improving the concept of treatment of diseases of the gastrointestinal tract and liver, which is accompanied by dysfunction of the hepatobiliary system and intestines, was the search and development of herbal remedies with hepatoprotective effect with a laxative effect and concomitant prebiotic activity.

Aim of the research: to study the antimicrobial and prebiotic properties of the studied phytosubstance obtained from the fruits of Prunus domestica.

Materials and methods. The study of the antimicrobial activity of the test sample of plum fruit extract with fibers was performed in vitro by the method of multiple serial dilutions. The reference strains of the following microorganisms were used as the microbiological model: S. aureus-ATCC-25923, E. coli-ATCC-25922, P. aéruginosa-ATCC-27853, B. subtilis-ATCC-6633, C. albicans-ATCC-885653.

The determination of the prebiotic properties of the studied sample was carried out by the method of cultivation of bacteria Bifidobacterium bifidum No. 1 and L. rhamnosus R0011 ND on nutrient media, which included the composition of plum fruit with fibers.

Results. Studies on the antimicrobial activity of plum fruit extract with fibers showed that the studied phytosubstance does not reveal antagonistic properties both with respect to gram-positive and gram-negative bacteria, as well as in the case of Candida fungi. The results of studies on the study of the prebiotic properties of the studied extract confirmed the improvement of the main physiological parameters of the culture of bifidobacteria strain Bifidobacterium bifidum No. 1 and the strain lactobacillus L. rhamnosus R0011 ND when the fruit plums with fibers were added to the culture medium.

Conclusions. The conducted studies allowed to establish the prebiotic properties of the studied extract of plum fruits with fibers, which proves the promise of its use in complex therapy schemes for gastrointestinal patholog.

Keywords: hepatoprotective agent, fruits of Prunus domestica, bifidobacteria, lactobacilli, prebiotic properties, antimicrobial activity. 


\section{Introduction}

The concept of hepatoprotective agents' use is to minimize the effects of toxic agents on the liver. Most medicinal products and xenobiotics are converted into the liver and initiate the first phase of the enzyme detoxification reaction, which causes the formation of active metabolites (products of lipid peroxidation) and leads to membrane-disruptive effect. The key role of the destabilizing action of free radicals, products of lipid peroxidation in the pathogenesis of liver diseases was proved, and the possibility of their treatment with hepatoprotective drugs was substantiated. The promising direction of the pharmacotherapy of liver disease is the use of phytopreparations. The advantages of pharmacotherapy with herbal medicines are: the absence of toxic effects, the possibility of prolonged use, a significant range and "softness" of the effect, the optimal pharmacoeconomic component, the possibility to use in patient at all age groups $[1,2]$. Therefore, the Prunus Domestica attracted the interesting and unique chemical content of its fruits and its wide application in folk medicine was chosen as object for experimental research [3].

Prunus domestica (family Rosaceae) is widespread horticultural crops in Ukraine. It has a lot breeds and occupy the second place in quantity after apples, pears and cherries [4, 5]. Extracts of Prunus Domestica were firstly developed at the department of chemistry of nature substances of the National University of Pharmacy [6].

In the previous research the qualitative and quantitative chemical composition of the Prunus domestica were determined. It was shown the presence of organic acids (malate, citrate, chlorogenic, neoclonogenic and coffeic acids) anthocyanins, rutin, gallic acid, sugars, dietary fibers and hydroxy-cinnamic acids in the raw material $[7,8]$.

According to literature data, Prunus domestica fruits are widely used in the treatment of diseases of the gastrointestinal tract (constipation and intestinal atony, bile stains, liver disease). Fruits of Prunus domestica are a source of polyphenolic compounds with a wide range of effects. The extract from the Prunus domestica fruits decreases the content of cholesterol in the blood, inhibits the development of tumors of the rectum by inhibiting phosphorylation and expression of protein kinase B/Akt. There is evidence of the effectiveness of the use of Prunus domestica extracts in the prevention and treatment of liver cancer. Flavonoids presented in Prunus domestica can be used in the treatment of hepatitis C [9].

The most active extract and its maximum effective dose were detected as a result of the pharmacological screening studies in the research of the laxative and hepatoprotective properties of extracts from Prunus domestica $[10,11]$. Characterizing the chemical composition of the fruits of the plum and taking into account the indications for use in diseases of the gastrointestinal tract, one should understand the prospects of using the drug on the basis of the fruit extract of the plum in diseases of infectious nature. Today, the use of antimicrobial drugs is often accompanied by various side effects, including the development of dysbiosis [12]. The basis of this syndrome is the imbalance of the microbiota of the intestine [13]. Among the autochthonous microorganisms, the main role belongs to bifidobacteria and lactobacilli $[14,15]$. It is difficult to overestimate the role of these microorganisms in the function of the human body [16]. Therefore, it was relevant to study the antimicrobial activity and prebiotics properties of the extract of fruit plum with fibers.

\section{Aim of research}

The purpose of these researches were to study the antimicrobial activity and the effect of the plum balance herbal substance on the growth of bifidobacteria and lactobacillus.

\section{Materials and Methods}

\section{1. Experimental procedures}

\section{1. 1. Study of antimicrobial activity}

Determination of antimicrobial activity was carried out by the method of serial dilutions, the essence of which consists in conducting two-fold dilutions of a test sample in $2 \mathrm{ml}$ of meat-pepton broth (MPB environment No. 1) (only 10 samples). It was used a separate pipette for each 
dilution. After that, $0.2 \mathrm{ml}$ of microbial curvature of a test strain with an appropriate number of microbial cells was injected into each test tube. Additionally, controls were prepared: 2 test tubes with $2 \mathrm{ml}$ of used medium in each - control of the medium; 2 test tubes of $2 \mathrm{ml}$ of the medium to which $0.2 \mathrm{ml}$ of microbial curvature of the test strain was also introduced to control the growth of the test microorganism.

As a microbiological model, were used the strains of the following microorganisms: $S$. aureus-ATCC-25923, E. coli-ATCC-25922, P. aeruginosa-ATCC-27853, B. subtilis-ATCC-6633, C. albicans-ATCC- 885653.

Inoculum of the bacterial and fungal cultures were prepared according to the optical turbidity standard of $0,5 \mathrm{IU}$ from a daily agar culture. The suspension of microorganisms (the microbial load $-150 \cdot 10^{6}$ microbes per $1 \mathrm{~mL}$ ) was transferred into the experimental solutions of extract of fruit plum with fibers and the reference test-tubes. The test-tubes containing bacterial cultures were kept in thermostat for $24 \mathrm{~h}$ at $37{ }^{\circ} \mathrm{C}$, and test-tubes containing the cultures were kept in thermostat for $48 \mathrm{~h}$. at $25{ }^{\circ} \mathrm{C}$. The lowest concentration of the experimental substation under research, at which any turbidity (growth of microorganisms) was not observed, was taken as the Minimum Inhibitory Concentration (MIC) value.

In the control of the growth of the test microorganism, the growth of microorganisms should be observed; the control of the environment should be sterile [17].

\section{2. Experimental procedures}

\section{2. 1. Study of prebiotic properties}

In order to confirm the prebiotic properties of the dry extract of fiber plums, cultivation of bacteria Bifidobacterium bifidum No. 1 and Lactobacillus rhamnosus R0011 ND in vitro was performed on media with the test sample.

In experiments, bacillus preparations were used, which consisted of B. bifidum and Lactobacillus rhamnosus. The preparation "Bifidobacterium dry" was pre-dissolved in the medium and activated at a temperature of $37-38^{\circ} \mathrm{C}$ for 24 hours. Then, this preparation was introduced into the nutrient medium prepared for cultivation in the range of 5 doses per 1 liter of medium. The cultivation of microorganisms was carried out under anaerobic conditions on a modified Bluraux medium. To determine the effectiveness of the action on the growth of B. bifidum microorganisms, the fruit plum extract with fibers (EPC) was included in the nutrient medium.

Cell counting of bifidobacteria was performed on fixed stained smears using the Vinogradsky-Shulgin-Brig method [17].

The drug "Lacidophil" was pre-dissolved in MRS-1 medium ( $\mathrm{pH} 6.7 \pm 0.1$ ) and reconstituted at $37 \pm 0.5{ }^{\circ} \mathrm{C}$ for 24 hours. Subsequently, sequencing was performed on media of MRS-2 ( $\mathrm{pH} 7,3 \pm 0.1)$ and MRS-4 ( $\mathrm{pH} 7.9 \pm 0.1)$. Then, in a quantity of $10 \%$, the culture was introduced into the prepared medium of KD-5. To determine the effectiveness of the action on the growth of L. rhamnosus R0011 ND microorganisms in the nutrient medium included EPRV. The number of live bacteria was determined by counting colonies on the MRS-4 medium [18].

The experiments were carried out in 3 times biological repeats.

Microscopy of cultures was performed using a Granum light microscope. According to the basic morphological characteristics, the declared cultures of bifidobacteria and lactobacilli corresponded to the established standards $[19,20]$.

\section{Results of research}

The first stage of research was the determination of the antimicrobial activity of the studied extract of plum fruits with fibers. Screening data is presented in Table $\mathbf{1 .}$

To solve these problems, the next stage of research was the study of prebiotic properties. For this study, the bacterial strains that are representatives of the human intestinal microbiota, $B$. bifidum and L. rhamnosus, were introduced into the screening. The experimental data are shown in Table 2, 3. 
Table 1

The results of the study of antimicrobial activity

\begin{tabular}{cccccc}
\hline \multirow{2}{*}{ Sample } & \multicolumn{5}{c}{ MIC, $\mathbf{m g} / \mathbf{m L}$} \\
\cline { 2 - 6 } & $\begin{array}{c}\text { S.aureus- } \\
\text { ATCC-25923 }\end{array}$ & $\begin{array}{c}\text { E.coli- } \\
\text { ATCC-25922 }\end{array}$ & $\begin{array}{c}\text { P. aeruginosa- } \\
\text { ATCC-27853 }\end{array}$ & $\begin{array}{c}\text { B. subtilis- } \\
\text { ATCC-6633 }\end{array}$ & $\begin{array}{c}\text { C. albicans- } \\
\text { ATCC- 885653 }\end{array}$ \\
\hline $\begin{array}{c}\text { Fruit extract of } \\
\text { Prunus domestica }\end{array}$ & growth & growth & growth & growth & growth
\end{tabular}

Table 2

Results of quantitative determination of bifidobacteria

\begin{tabular}{cc}
\hline Samples & CFU/mL \\
\hline Nutrient medium with EFPF & $10^{11}$ \\
Control (nutrient medium without additional components) & $10^{10}$
\end{tabular}

Table 3

Results of quantitative determination of lactobacilli

\begin{tabular}{cc}
\hline Samples & Number of living bacteria, billion microbial cells \\
\hline Nutrient medium with EFPF & $6.2 \pm 0.2$ \\
Control (nutrient medium without additional components) & $5.9 \pm 0.2$
\end{tabular}

\section{Discussion of researches}

Antimicrobial activity is one of the positive criteria for the effectiveness of drugs in the pharmacotherapy of diseases of the gastrointestinal tract. Taking into account the literature data on the chemical composition of plum fruit, special attention is paid to the rather high content of organic acids (apple, amber, etc.). Given the insignificant content of salicylic acid, one of the tasks of the work was to study the antimicrobial activity of the investigated extract.

The antimicrobial activity of extract of fruit plum with fibers in vitro was studied in accordance with the requirements of the State Pharmacopoeia of Ukraine (ed.1) by the double serial dilution method in the liquid growth medium. The extract of fruit plum with fibers were tested against Pharmacopoeia strains of gram negative (E. coli ATCC 25922, P. aeruginosa-ATCC-27853) and gram positive ( $S$. aureus ATCC 25923, B. subtilis-ATCC-6633) bacteria, as well as against the fungal strain of Candida albicans (ATCC 885653).

The results of the researches showed that the extract of fruit plum with fibers did not show antimicrobial activity in relation to gram-positive (S. aureus, B. subtilis), gram-negative (E. coli, P. aeruginosa) and fungi of the genus Candida. Data are given in Table $\mathbf{1 .}$

Considering the chemical composition of the fruits of Prunus domestica, represented by organic substances with weak acidic properties, literature data on the effectiveness of the use of phyto-raw materials (plum-based preparations), and an assumption was made about the possible prebiotic properties of the obtained extract.

Studies have shown that bifidobacteria of strain Bifidobacterium bifidum No.1 have shown good growth rates in the experimental nutrient medium. Analysis of the control bifidobacteria showed that the introduction of the test substance into the nutrient medium was accompanied by an increase in the number of bacteria $(1011 \mathrm{CFU} / \mathrm{ml})$. In the control, quantitative growth indicators of bifidobacteria were detected at a level of 1010 CFU/ml. Data are given in Table 2.

Morphological signs of bacteria Bifidobacterium bifidum No.1 with growth in the experimental nutrient medium were preserved and corresponded to the literature.

In determining the impact of extract of fruit plum with fibers on the growth of lactobacilli showed that the introduction of experimental substance positively affects the growth of culture of lactobacilli. Analysis of the results of counting cells of L. rhamnosus R0011 ND on MRS-4 me- 
dium showed that in comparison with the control, the amount of lactobacilli when introduced into the nutrient medium of the additional component (EFPF) was accompanied by an increase in the number of microbial cells in almost 1.1 times. Data are given in Table 3.

Establishing the prebiotic properties of an experienced, first-time-received sample of plum fruit extract with fibers requires further research both in vitro and in vivo. Positively assessing the results obtained, one should study the intensity of metabolic processes of bifidobacteria and lactobacilli on media containing Prunus domestica fruit extract as an additional prebiotic component.

Analysis of literature data on the study of the antimicrobial activity of plum extracts showed that some of its objects (leaves, fruits) are characterized by the presence of antibacterial properties. In particular, extracts obtained from the leaves of Prunus salicina revealed antimicrobial activity due to the presence of polyphenols in the chemical composition [21]. As for extracts obtained from plum fruits (water extract from the cake), bacteriostatic activity has been established against some pathogenic microorganisms, which is mediated by the presence in the chemical composition of a large content of flavonol [22]. Evaluating the results of the primary antimicrobial screening of the dry extract from fruit of the Prunus domestica we are studying, it should be noted that the low content of flavonol derivatives explains the absence of antibacterial action and its low toxicity [23].

\section{Conclusions}

1. It is proved that the phytosubstance does not reveal antimicrobial activity in relation to reference cultures $S$. aureus, E. coli, B. subtilis, P. aeruginosa, C. albicans.

2. As a result of the research, it was established that the cultivation of bifidobacteria Bifidobacterium bifidum No. 1 and lactobacillus L. rhamnosus R0011 ND in vitro conditions on a medium with an extract of pollen extracts with fibers showed good growth rates and biomass accumulation.

3. The studies have shown that the presence of prebiotic properties in the fruit extract of plum with fibers, accompanied by the intensification of growth of both bifidobacteria and lactobacilli.

4. It is proved that the studied extract from Prunus domestica fruits with fibers is promising for use in complex therapy schemes for gastrointestinal pathology.

\section{References}

[1] Ubeeva, E. A., Nickolaev, S. M., Ubeeva, I. P. (2017). The main directions of phytotherapy of liver diseases. Vestnik Medicine and Pharmacy, 3, 3-9.

[2] Ubeeva, I. P., Nickolaev, S. M., Botoeva, E. A. (2009). Herbal medicine for diseases of the hepatobiliary system. Irkutsk: RIO IGIUAs, 109.

[3] Grodinsky, A. M. (Ed.) (1992). Medicinal plants: Encyclopedic reference book. Kyiv: "Ukrainian Encyclopedia" Publishing House named after M. P. Bazhan, Ukr. Production and Commercial Center "Olympus", 544.

[4] Chernykh, V. P. (Ed.) (2010). Pharmaceutical encyclopedia. Kyiv: MORION, 1952.

[5] The State Pharmacopoeia of Ukraine (2008). State Enterprise "Ukrainian scientific Pharmacopoeia center". Kharkiv: RIREG, 620 p.

[6] Basim, M. S., Lenchik, L. V., Caidov, N. B. (2017). Prunus Domestica fruits - perspective row for drugs development. Nauka i innovaciya. Seriya estestvennyh nauk, 4, 42-48.

[7] Upyr, T., Basim Mohammed, S., Bashar, A.-J. A. S., Lenchyk, L., Senyuk, I., Kyslychenko, V. (2018). Phytochemical and pharmacological study of polysaccharide complexes of prunus domestica fruit. ScienceRise: Pharmaceutical Science, 3 (13), 32-37. doi: http://doi.org/10.15587/2519-4852.2018.135825

[8] Celik, F., Gundogdu, M., Alp, S., Muradoglu, F., Ercişli, S., Gecer, M. K., Canan, I. (2017). Determination of phenolic compounds, antioxidant capacity and organic acids contents of Prunus domestica L., Prunus cerasifera Ehrh. and Prunus spinosa L. fruits by HPLC. Acta Chromatographica, 29 (4), 507-510. doi: http://doi.org/10.1556/1326.2017.00327

[9] Kayano, S., Kikuzaki, H., Fukutsuka, N., Mitani, T., Nakatani, N. (2002). Antioxidant Activity of Prune (Prunus domesticaL.) Constituents and a New Synergist. Journal of Agricultural and Food Chemistry, 50 (13), 3708-3712. doi: http://doi.org/10.1021/jf0200164 
[10] Senyuk, I. V., Bashar, A.-S. J., Lenchyk, L. V. (2017). Investigation of different substances catharic properties made from Prunus domestica. Ukrainian biopharmaceutical journal, 5 (52), 21-25. doi: http:// doi.org/10.24959/ubphj.17.134

[11] Senjuk, I. V., Al Sahlani, B. J., Basim, M. S. (2018) Study of hepatoprotective effect of extracts from Prunus domestica fruits. Pharmaceutical review, 4 (48), 57-61. doi: http://doi.org/10.11603/23120967.2018.4.9692

[12] Tochilina, O. A., Chastoedova, I. A. (2013). Intestinal dysbiosis and enzyme-excretory function in young children after use antibiotics and methods of correction. Human ecology, 9, 44-48.

[13] Gibson, G. R., Probert, H. M., Loo, J. V., Rastall, R. A., Roberfroid, M. B. (2004). Dietary modulation of the human colonic microbiota: updating the concept of prebiotics. Nutrition Research Reviews, 17 (2), 259-275. doi: http://doi.org/10.1079/nrr200479

[14] Astashkina, A. P. (2010). Modern views on the biological role of bifidobacteria and lactobacilli. Vestnik VGU. Series: Chemistry. Biology, Pharmacy, 1, 133-139.

[15] Anderson, R. C., Cookson, A. L., McNabb, W. C., Park, Z., McCann, M. J., Kelly, W. J., Roy, N. C. (2010). Lactobacillus plantarum MB452 enhances the function of the intestinal barrier by increasing the expression levels of genes involved in tight junction formation. BMC Microbiology, 10 (1), 316. doi: http://doi.org/10.1186/1471-2180-10-316

[16] Lifshitz, L., Zakharova, I. N., Dmitrieva, Y. A. (2017). Effect of intestinal microbiome in norm and pathology on human health. Medical Council, 1, 155-159. doi: http://doi.org/10.21518/2079701x-2017-1-155-159

[17] Volyansky, Yu. L., Gritsenko, I. S., Shirobokov, V. P. et. al. (2004). Study of specific activity of antimicrobial drugs. Kyiv, 40.

[18] Cheremushkina, I. V., Glushchenko, A. S., Anohina, E. P., Chigirina, N. A., Cherenkov D. A., Slepokurov, A. A. et. al. (2010). Biotechnology mannozosoderzhaschih hydrolysates and study of prebiotic properties of mannose. Biotechnology, 5, 56-61.

[19] Holt, J., Krieg, N., Smith, P., Staley, G., Williams, C. (1997). The determinant of Berdji bacteria. Vol. 1. Moscow: Mir, 432.

[20] Holt, J., Krieg, N., Smith, P., Staley, G., Williams, C. (1997). The determinant of Berdji bacteria. Vol. 2. Moscow: Mir, 359.

[21] Delgado-Adámez, J., Fernández-León, M. F., Velardo-Micharet, B., González-Gómez, D. (2012). In vitro assays of the antibacterial and antioxidant activity of aqueous leaf extracts from different Prunus salicina Lindl. cultivars. Food and Chemical Toxicology, 50 (7), 2481-2486. doi: http://doi.org/10.1016/j.fct. 2012.02.024

[22] Sójka, M., Kołodziejczyk, K., Milala, J., Abadias, M., Viñas, I., Guyot, S., Baron, A. (2015). Composition and properties of the polyphenolic extracts obtained from industrial plum pomaces. Journal of Functional Foods, 12, 168-178. doi: http://doi.org/10.1016/j.jff.2014.11.015

[23] Kravchenko, V. M., Sahlani, B. J. A., Senyuk, I. V. (2018). Investigation of acute toxicity of plum home fruit extracts. Prospects for the development of medicine in EU countries and Ukraine. Wloclawek, 109-112. 